\title{
START TO END SIMULATIONS OF THE ERL PROTOTYPE AT DARESBURY LABORATORY
}

\author{
C. Gerth*, M. Bowler, B. Muratori, H.L. Owen, N.R. Thompson, \\ ASTeC, Daresbury Laboratory, Warrington WA4 4AD, UK \\ B. Faatz, DESY, Notkestraße 85, D-22603 Hamburg, Germany \\ B.W.J. McNeil, University of Strathclyde, Glasgow, G2 0NG, Scotland
}

\begin{abstract}
Daresbury Laboratory is currently building an Energy Recovery Linac Prototype (ERLP) that will serve as a research and development facility for the study of beam dynamics and accelerator technology important to the design and construction of the proposed 4th Generation Light Source (4GLS) project. Two major objectives of the ERLP are the demonstration of energy recovery and of energy recovery from a beam disrupted by an FEL interaction as supplied by an infrared oscillator system. In this paper we present start-to-end simulations of the ERLP including such an FEL interaction. The beam dynamics in the highbrightness injector, which consists of a DC photocathode gun and a superconducting booster, have been modelled using the particle tracking code ASTRA. After the booster the particles have been tracked with the code elegant. The 3D code GENESIS 1.3 was used to model the FEL interaction with the electron beam at $35 \mathrm{MeV}$. A brief summary of impedance and wakefield calculations for the whole machine is also given.
\end{abstract}

\section{INTRODUCTION}

The performance of a free-electron laser (FEL) depends crucially on the electron beam parameters. While analytical calculations can give an estimate of the expected performance, numerical start-to-end (S2E) simulations are required to account for various aspects of beam dynamics during the generation, transport and compression of the beam (see for example [1]). FELs based on the Energy Recovery Linac (ERL) concept have a distinct advantage in terms of RF power and beam dump requirements. However, another aspect becomes important for S2E simulations: the electron beam, which may have a large energy spread induced by the FEL process, needs to be recirculated for deceleration and then transported into the beam dump [2].

Daresbury Laboratory are currently building an ERL Prototype [3] which will operate at a beam energy of $35 \mathrm{MeV}$ and drive an infra-red oscillator FEL. In this paper we present the results of S2E simulations for the ERLP including the FEL. To account for space charge effects, ASTRA [4] was used for the modelling of the low energy part $(350 \mathrm{keV})$ of the injector from the cathode to the booster. The beam was tracked with elegant [5] from the booster $(8.35 \mathrm{MeV})$ to the main linac $(35 \mathrm{MeV})$ and then to the

\footnotetext{
* now at DESY: Christopher.Gerth@ desy.de
}

FEL. The FEL interaction was modelled with GENESIS, and elegant was used to transport the beam back to the linac for energy recovery and then to the beam dump.

\section{INJECTOR}

The injector consists of a high-average current DC photocathode gun, a booster and a transfer line to the main linac. The DC photocathode gun is a replica of the $500 \mathrm{kV}$ Jefferson Lab gun [6] and will operate at a nominal accelerating voltage of $350 \mathrm{kV}$ and bunch charge of $80 \mathrm{pC}$. Electrons will be generated at a GaAs photocathode by the frequency-doubled light $(532 \mathrm{~nm})$ of a mode-locked $\mathrm{Nd}: \mathrm{YVO}_{4}$ laser with an oscillator frequency of $81.25 \mathrm{MHz}$. Two solenoids are used for transverse focusing and emittance compensation, and a normal-conducting single-cell buncher cavity is utilised to decrease the bunch length from the GaAs cathode. The buncher cavity will be operated at $1.3 \mathrm{GHz}$ and is based on the design employed at the ELBE facility. Electrons are accelerated to an energy of $8.35 \mathrm{MeV}$ in the booster, which consists of two super-conducting 9cell TESLA-type cavities operated at $1.3 \mathrm{GHz}$; the cryomodule design is based on the design of the ELBE linac [7]. The layout of the ERLP injector is shown in Fig. 1 and a description of the design can be found in Ref. [8].

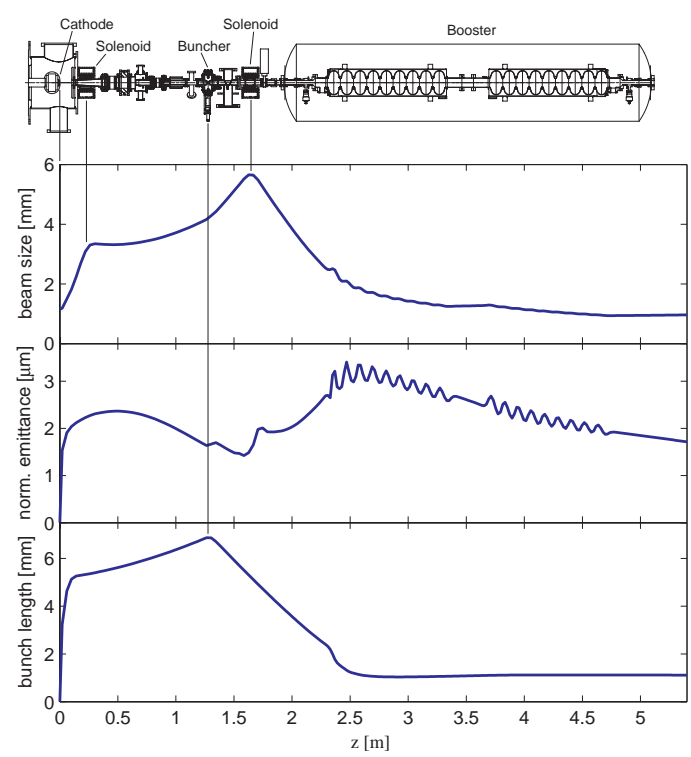

Figure 1: Layout of the ERLP injector and evolution of the beam size, norm. emittance and bunch length (all rms). 
The transverse properties of the electron bunch at the cathode are determined by the cathode laser whereas the longitudinal profile is dominated by the GaAs cathode for short laser pulses, due to the rather long response time of GaAs. For simulation a longitudinal Gaussian distribution of $20 \mathrm{ps} \mathrm{rms} \mathrm{length} \mathrm{was} \mathrm{assumed.} \mathrm{The} \mathrm{transverse} \mathrm{distribu-}$ tion was chosen to be Gaussian with an rms beam size $\sigma_{r}$ $=1.25 \mathrm{~mm}$ truncated at $\pm 2 \sigma_{r}$. Results for the evolution of the rms values of the beam size, normalised emittance and bunch length are shown in Fig. 1 for 250k macro-particles.

A transfer line takes the beam to the main linac where it is merged with the full energy $(35 \mathrm{MeV})$ single-pass recirculated beam. 3D simulations using GPT [9] indicate a moderate emittance growth, which is taken into account in the elegant S2E simulations by using appropriate RF phase and sextupole settings (see below).

\section{BEAM TRANSPORT SYSTEM}

Electrons from the injector are accelerated to $35 \mathrm{MeV}$ in the super-conducting main linac, which is identical to the booster and composed of two 9-cell TESLA-type cavities. Two $180^{\circ}$ triple-bend achromat (TBA) arcs are used to recirculate the beam to the main linac where the electrons are decelerated to their injection energy and subsequently dumped. A 4-dipole chicane provides bunch compression upstream of the wiggler and bypasses the upstream FEL mirror.

The minimum bunch length is required within the wiggler. The compression chicane has a static $R_{56}^{C}$ of $0.28 \mathrm{~m}$, which requires an off-crest phase of about $\varphi_{\mathrm{rf}} \simeq 9^{\circ}$ in the main linac for full compression. The TBA arcs are able to provide a variable large negative $R_{56}$. In the nominal setup the first arc is set to $R_{56}^{A 1}=0$ whilst the second is tuned to $R_{56}^{A 2}=-R_{56}^{C}$ to decompress the bunch. The sextupoles in the first arc can be used to linearise the lowest-order curvature induced by the sinusoidal RF during acceleration, by varying $T_{566}$. The effect of the sextupoles on is demonstrated in Figure 2 for an example case. The sextupoles in the second arc may be used to minimise the energy spread after deceleration for optimal energy recovery and extraction to the beam dump.

Results of the S2E simulations for the longitudinal phase space and bunch profile at 4 different locations in the ERLP are shown in Fig. 3. The ASTRA particle distribution from
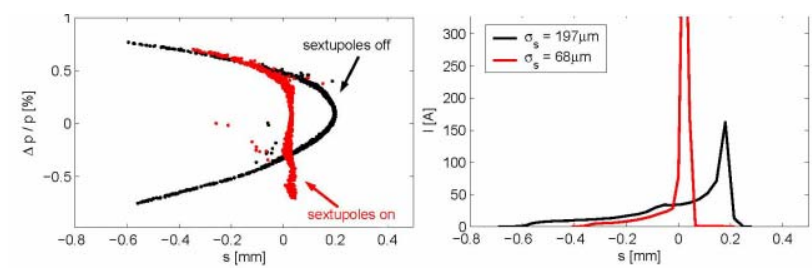

Figure 2: Comparison of longitudinal phase space after the compressor chicane with sextupoles turned on $\left(100 \mathrm{~m}^{-3}\right)$ and off.
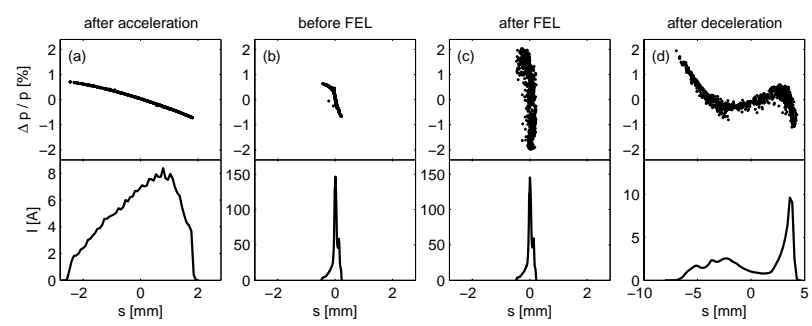

Figure 3: Longitudinal phase space and bunch profile at different locations in the ERLP: (a) after off-crest acceleration by $\varphi_{\mathrm{rf}}=7.8^{\circ}$; (b) after the compressor chicane with the sextupoles set to $100 \mathrm{~m}^{-3}$; (c) after FEL interaction; (d) after energy recovery.

the injector modelling was used as an input for elegant, and the beam was tracked from the exit of the booster to the wiggler entrance; mad8 was used to match the lattice functions. The bunch was not fully compressed in the S2E simulation (Fig. 3(b)): the RF phase $\varphi_{\mathrm{RF}}$ and sextupole settings were chosen to approximate the expected beam parameters at the wiggler entrance, thus simulating the effects of the neglected space charge in the injector to linac transport. The FEL interaction induces a large energy spread (see Fig. 3), and was modelled with GENESIS 1.3; this is described in more detail in the next section. The particle distribution was then converted back to elegant and tracked to the beam dump. When the second arc is set to $R_{56}^{A 2}=-R_{56}^{C}$ the deceleration phase is given by $\varphi_{\mathrm{RF}}+\pi$; however, to return exactly to the injection energy after deceleration, the RF phase must be reduced slightly to account for the mean energy loss of about $0.8 \%$ in the FEL process. Apertures were included in the elegant tracking, which were chosen to be $10 \%$ smaller than the envisaged vacuum chamber dimensions to approximately model misalignments estimate using mad8: no particles were lost during recirculation even with the sextupoles turned off in the second arc. Preliminary calculations of resistive-wall and bellows wakefield effects indicate that the induced energy spread from these impedances is small.

\section{FEL}

The wiggler has been supplied on loan from Jefferson Laboratory, and is a planar device with 40 periods of length $27 \mathrm{~mm}$. The magnet arrays are vertically aligned, giving focusing in the horizontal plane. The matched beam conditions in transverse phase space are thus a waist in the horizontal plane at the wiggler entrance and a waist in the vertical plane at the wiggler centre. This corresponds to a desired $\beta_{x}$ at the wiggler entrance of $0.5 \mathrm{~m}$, and $\alpha_{y}=1.75$ and $\beta_{y}=1.25 \mathrm{~m}$ in the vertical plane to give the minimal vertical beam radius averaged along the wiggler.

The optical cavity length is $D=9.224 \mathrm{~m}$ with the wiggler positioned at the cavity centre; the mirror radii of curvature $R_{1}$ and $R_{2}$ are chosen to give a near-concentric cavity with an optical waist at the wiggler centre. The Rayleigh 
length is $0.75 \mathrm{~m}$ compared to a wiggler length of $1.08 \mathrm{~m}$ - the optimum Rayleigh length for FEL coupling would be less than this but would drive the cavity towards instability; the cavity stability is given by $g_{1} \cdot g_{2}=0.9$, with $g_{1}=1-D / R_{1}$ and $g_{2}=1-D / R_{2}$.

The FEL process was modelled with GENESIS as follows: first, the projected rms values of the tracked particle distribution were calculated, and the predicted performance of the FEL was estimated with analytical formulae and with GENESIS in steady-state mode (FEL wavelength, intracavity power, etc.). These results and the SDDS toolkit program elegant2genesis were then used to generate a GENESIS input file, the code being run in time-dependent mode with a seed power given by the analytic estimate of the intra-cavity peak power at saturation of $\approx 80 \mathrm{MW}$. This seed power is approximated as a uniform intensity over the entire electron bunch. Although this is not an exact representation of the pulse structure in a cavity it should approximate reasonably well the energy spread induced by the FEL interaction.

The utility code elegant2genesis discretises the macroparticle distribution supplied by elegant into radiation wavelength slices (here $4.4 \mu \mathrm{m}$ ) and calculates the relevant GENESIS input parameters. The charge of each slice is proportional to the number of macro-particles it contains. These parameters are then applied to form a GENESIS macro-particle distribution with a constant number of particles for each slice (typically 8192). To convert the GENESIS output file back to an elegant input file, the number of particles in each slice should again be made proportional to the slice charge. This is achieved by randomly sampling the GENESIS particles to give the required number of elegant macro-particles. Care should be taken when converting complex particle distributions using elegant2genesis as inhomogeneous distributions may be inadvertently simplified as the script only calculates the mean and rms values for each slice; this is important in cases where the bunch tails occur in the same slice but occupy two separate energy bands.

The energy profiles of the electron bunch before and after the FEL process are compared in Fig. 4 for a seed power of $80 \mathrm{MW}$. The mean energy loss in the simulation is $0.8 \%$ which is in good agreement with analytical predictions. The return arc must have an energy acceptance sufficient to transport all the electrons. Assuming a Gaussian distribution a range of $\pm 3 \sigma_{E}$ contains $99.7 \%$ of the electrons, so an estimate of the full FEL exhaust energy spread is given by $6 \sigma_{E}$. A full energy spread of $\approx 4 \%$ is predicted by one-dimensional steady-state code for the given parameters which is in good agreement with the GENESIS results. (Fig. 4).

\section{SUMMARY AND OUTLOOK}

S2E simulations of an ERL including both an FEL interaction and energy recovery have been performed. The particle tracking codes ASTRA and elegant were used for

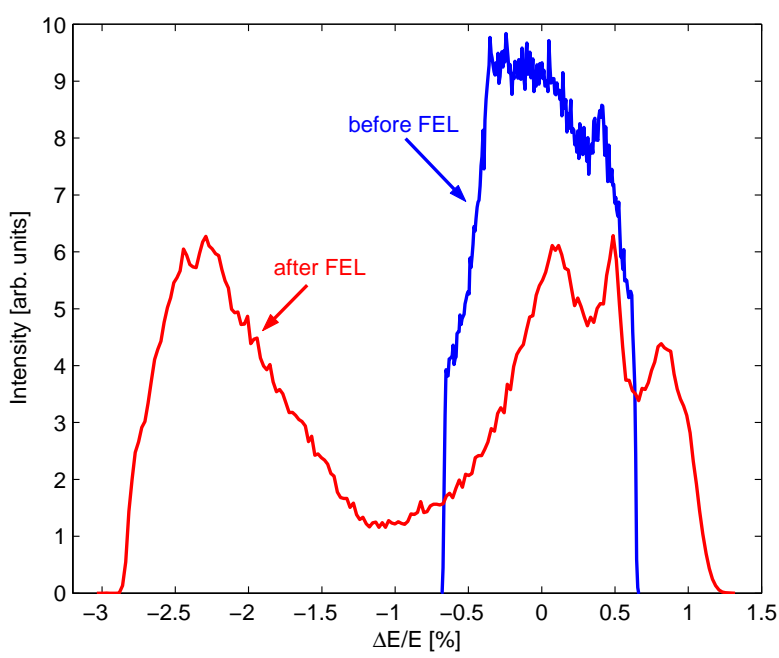

Figure 4: Results of GENESIS simulation: energy spread induced by the FEL interaction with $80 \mathrm{MW}$ seed beam (data taken from Figs. 3(b) and (c)). Energies are shown relative to the mean input energy.

particle transport. The FEL oscillator was modelled as a seeded single pass amplifier configuration with a seed power equivalent to the estimated intra-cavity peak power at saturation.

\section{REFERENCES}

[1] M. Dohlus et al., "Start-To-End Simulations of SASE FEL at the TESLA Test Facility, Phase I: comparison with experimental results", Nucl. Instr. and Meth. A 528, 448, 2004.

[2] P. Piot et al., "Longitudinal phase space manipulation in energy recovering linac-driven free-electron lasers", Phys. Rev. ST-AB 6, 030702, 2003.

[3] M.W. Poole, E.A. Seddon, "4GLS and the Energy Recovery Linac Prototype Project at Daresbury Laboratory", These Proceedings.

[4] K. Flöttmann, "ASTRA User Manual”, September 18, 2000. http//www.desy.de/ mpyflo.

[5] M. Borland, "elegant: A Flexible SDDS-Compliant Code for Accelerator Simulation ”, APS LS-287, September 2000.

[6] T. Siggins et al., "Performance of a DC GaAs photocathode gun for the Jefferson lab FEL", Nucl. Instr. and Meth. A 475 (2001) 549.

[7] A. Büchner et al., "The ELBE-Project at DresdenRossendorf”, EPAC'00, Vienna, June 2000.

[8] C. Gerth, F.E. Hannon, "Injector Design for the 4GLS Energy Recovery Linac Prototype”, EPAC’04, Lucerne, July 2004.

[9] B. Muratori et al., "Space Charge effects for the ERL Prototype", These Proceedings. 